\title{
Visualizing Business Ecosystems: Applying a Collaborative Modelling Process in Two Case Studies
}

\section{Anne Faber}

Department of Informatics

Technical University of Munich

Garching bei München, Deutschland

Email: anne.faber@tum.de

\section{Adrian Hernandez-Mendez}

Department of Informatics

Technical University of Munich

Garching bei München, Deutschland

Email: adrian.hernandez@tum.de

\section{Sven-Volker Rehm}

EM Strasbourg Business School, HuManiS (EA 7308)

Université de Strasbourg

Strasbourg Cédex, France

Email: sven.rehm@em-strasbourg.eu

\section{Florian Matthes}

Department of Informatics

Technical University of Munich

Garching bei München, Deutschland

Email: matthes@tum.de

\begin{abstract}
Business ecosystems are increasingly gaining relevance in research and practice. Because business ecosystems progressively change, enterprises are interested in analysing their ecosystem, to identify and address such changes. In order to gain a comprehensive picture of the business ecosystem, various stakeholders of the enterprise should be involved in the analysis process. We propose a collaborative approach to model and visualize the business ecosystem and we validate four central roles in the modelling process. The process consists of six steps, namely the definition of the business ecosystem focus, instantiation of the model, data collection, provision of tailored visualizations, collecting feedback and adapting the models, and using the visualization 'to tell a story'. In this paper, we report case studies of two companies that have instantiated ecosystem models.
\end{abstract}

Keywords Business Ecosystem, Collaborative Modelling, Visualization, Case Studies, Lessons Learned. 


\section{Introduction}

Undoubtedly, companies increasingly recognize the relevance of their complex business environment in which they develop, produce and distribute their services and products. This environment is often referred to as business ecosystem. Addressing the associated challenges and opportunities is a reality for most companies (Peltoniemi and Vuori 2004). The growing relevance of business ecosystems substantiates through the perceived shift of the competitive environment from single companies and their supply chains towards ecosystems competing against each other (Bosch 2016).

Thereby, a business ecosystem extends the classic supply chain, consisting of suppliers and customer, by also including other entities within the business environment of the enterprise. We define business ecosystems as the holistic environment of a company covering current and potential future business partners, customers, suppliers, competitors, regulatory institutions, and innovative start-ups. It exhibits a high dynamic as continuously entities enter and leave the ecosystem. Peltoniemi and Vuori (2004) provide a comprehensive definition of business ecosystems. Analogously to the metaphor of a biological ecosystem, which served as a basis for the initial definition of business ecosystems (Moore 1997), the economic success of an enterprise can therefore depend on the health and ability to evolve their business ecosystem. The role of the enterprise within its ecosystem can range from a keystone to a niche player, with varying level of influence on the overall health of the ecosystem (Iansiti and Levien 2004).

Due to the influence on the economic success of the enterprise and the dynamic characteristics, enterprises increasingly realize the need to analyse their business ecosystem continuously, in order to identify and address changes within their ecosystem (Basole et al. 2016), adapt own business activities accordingly and to "learn what makes the environment tick" (Porter 1979).

Visualizations of business ecosystems have proven to support decision makers in their ecosystem related tasks (Basole et al. 2016; Huhtamaki and Rubens 2016, Evans and Basole 2016). Visualizing data can help to derive value from ecosystem data, e.g., in order to spot anomalies, identify keystone and niche players of the ecosystem, or recognize change patterns and trends (Vartak et al. 2016).

Ecosystem data is large and heterogeneous (Basole et al. 2015), ranging from technology-related data about applied standards and platforms to use, to market information and legal regulations. When focusing on business aspects of the company's business ecosystem, information about business partners, competitors, interesting start-ups and their strategies, partnerships and offered solutions, and cooperative initiatives become relevant (Faber et al. 2018). Data comprising this information can come from various sources, such as existing databases, newspaper articles or blogs addressing recent developments within the ecosystem, but also company and institutional web presences and publications. The issues associated to data collection in emergent business ecosystems are not yet resolved (Iyer and Basole 2016; Hao et al. 2015) which poses particular challenges for utilizing visualizations for ecosystem analysis or business development (Rehm et al. 2017).

In addition, not only various data sources but also stakeholders of several business units within the enterprise should be included in the business ecosystem analysis to involve diverse aspects and perspectives of the ecosystem. Collaborative modelling provides an approach to include a group of stakeholders into shared model creation and evaluation to improve the outcome of their cooperation (Dollmann et al. 2011). We thus aim at providing a collaborative approach to model and visualize business ecosystems from a company internal perspective. Thereby, we address the following research question:

\section{What are the challenges in collaborative approaches for modelling and visualizing business ecosystems and how does a model-driven approach address these challenges?}

Our contributions in this paper involve the description of 1) a process to initiate the modelling of business ecosystems, 2) important roles during the modelling process, and 3) lessons learned from two action research case studies we conducted with two companies targeting different business ecosystems.

The paper is structured as follows; in Section 2 we introduce related work of business ecosystem modelling, visualization and collaborative modelling targeting business ecosystems; in Section 3 the research methodology is described; this is followed by a description of the framework we used in both studies (Section 4). In Section 5, we present the two case studies in detail; and we summarize the lessons learned in Section 6. Finally, we provide a short discussion and an outlook (Section 7). 


\section{Related Work}

Business Ecosystem Modelling. Since the introduction of business ecosystems by James Moore in the mid-1990s, who defined it as a collection of interacting companies (Moore 1997), the concept has been widely studied and used in Management Science (Guittard et al. 2015). The initial definition was enriched describing the role of companies as "suppliers, distributors, outsourcing firms, makers of related products or services, technology providers, and a host of other organizations" (Iansiti and Levien 2004), all affecting business success and failure of companies active within the business ecosystem. Furthermore, business ecosystems constantly evolve, exhibiting a dynamic structure (Peltoniemi and Vuori 2004), with not only companies but also human actors, entering and leaving the ecosystem, which "are interconnected through a complex, global network of relationships" (Basole et al. 2015).

The Internet of Things (IoT) business ecosystem modelling gained researchers' attention, addressing business ecosystem design methods (Uchihira et al. 2016), presenting a framework to fully understand the complex IoT ecosystem. Current approaches further focus on frameworks to grasp the scope of ecosystem complexity (Iyer and Basole 2016), on visualizations to understand emerging structures and patterns (Iyer and Basole 2016; Leonardi 2011), or on policy contexts, e.g., smart city (Visnjic et al. 2016).

Recently, Sako (2018) defined three meta-characteristics of business ecosystems, sustainability, selfgovernance, and evolution, to contribute to a better distinction of the ecosystem concept from clusters or networks. Thereby, he focuses on "value-creating process (...) rather than an industrial sector".

Business Ecosystem Visualization. Visualizations of business ecosystems have proven to enable ecosystem stakeholders to take better-informed decisions (Basole et al. 2016; Huhtamaki and Rubens 2016; Evans and Basole 2016). Research addressing ecosystem visualizations has used data sets collected from commercial databases on business and economic data or drawn from social or business media (Basole et al. 2012; Basole et al. 2015).

Similar to the framework we used in our studies, Park et al. (2016) present a visual analytic system for analysis of a supply chain management ecosystem. The authors identify three salient design requirements: (1) to support multiple views in an integrated interface, (2) to enable interactive investigation of supply networks, and (3) to provide data-driven analytic capabilities. System users are enabled to interactively explore the ecosystem model using multiple views, all integrated in one user interface. In addition, data-driven analytics are provided. The system introduces five network layouts, which are force-directed, circular or chord diagram, tree map, matrix, and substrate-based layout. Thereby, all visualizations provide interactive features, such as clicking, dragging, hovering, and filtering. This work is based on extensive research in the area of modelling, visualizing and analyzing of business ecosystems (Basole et al. 2015; Park et al. 2016; Park and Basole 2016; Basole 2009a; Basole 2009b; Visnjic et al. 2016).

Collaborative Modelling of Business Ecosystems. Although collaborative modelling originated in the 7o's and has since increasingly gained relevance together with the increased need for collaboration amongst experts (Renger et al. 2008). Collaborative modelling has been applied to various research fields, such as business process modelling (Dollmann et al. 2011), enterprise architecture modelling (Roth et al. 2013), or group decision support system modelling (Liu and Zhang 2010), to name just a few. However, extant literature does not suggest collaborative processes that specifically address business ecosystem modelling and the instantiation of such models.

Roles essential for collaborative modelling have been identified as; the facilitator, the modeller, the process coach, the recorder, and the gatekeeper (Richardson and Andersen 1995), whereby these roles can be allocated to different persons - or several roles can be assigned to the same person (Renger et al. 2008). Richardson and Anderson (1995) describe the roles as, (a) facilitator, monitoring the group process and stimulating the model building effort; (b) modeller, focusing on the model outcome; (c) process coach, observing the process and the dynamics of the participants; (d) recorder, documenting the modelling process; and (e) gatekeeper, responsible for the process and major decision maker.

\section{Research Methodology}

We present results from two action research case studies with two different organizations. The work is part of a larger design science project, which we refer to as the Business Ecosystem Explorer (BEEx) and which is explain briefly in Section 4. In this design science project, we develop a tool to 
collaboratively model and visualize business ecosystems. The two action research case studies serve as evaluation studies for BEEx as design artefact.

We draw on the steps suggested by Yin (1994) for case study design and performed our study in seven consequent steps as illustrated in Figure 1. In this Section, we justify the design of our study and describe how it was followed to investigate a collaborative approach to model and visualize business ecosystems in two enterprise settings.

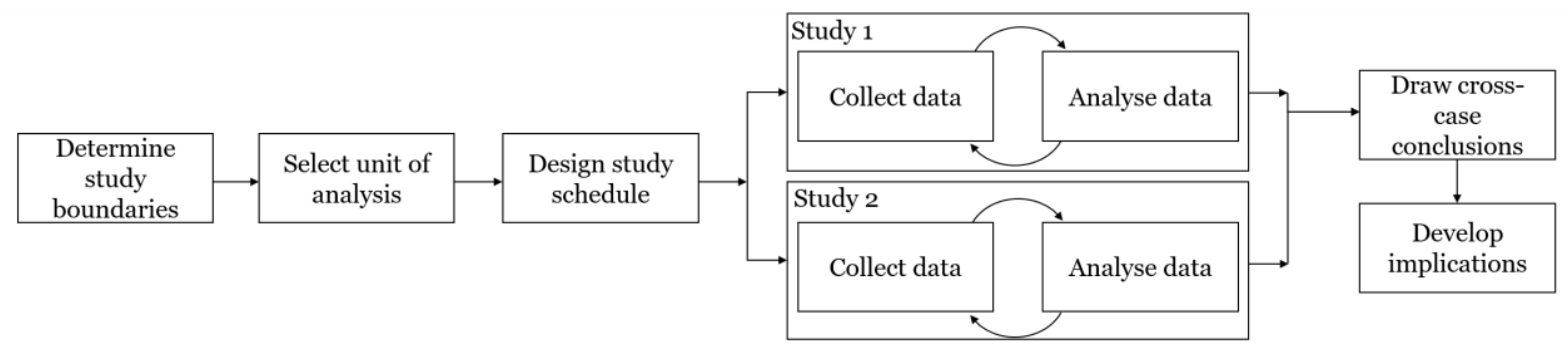

Figure 1: Multiple-case studies design steps (adapted from Yin (1994))

Determine Study Boundaries. Due to the lack of existing research and reports about a collaborative approach to model and visualize business ecosystems, in our studies we focus on the instantiation of such a process. We identified two companies, both with no business ecosystem model in place and interested in analysing a specific ecosystem.

Unit of Analysis. In the here presented work, we present results from two action research case studies. One organization is an automotive company, headquartered in Europe with approximately 120,000 employees. The other organization is a publishing company, headquartered in Europe with approximately 16,000 employees. Both organizations had a high interest in modelling and visualizing business ecosystems of their specific focus, one related to smart cities and the other to service and person landscape within their business area. For both, the ecosystem of focus was not yet modelled or visualized.

Design Study Schedule. With both organizations, several workshops were conducted in the period December 2017 to June 2018. For both studies, discussions about the study scheduled happened prior to the study period. In addition, previous work of the researchers was presented and discussed in advance of the study. We scheduled the workshops and meetings with the studies on an ad-hoc basis.

Data Collection. We follow Benbasat et al. (1987) to collect data through a) documentation, b) archival records, and c) direct observation. All involved stakeholders had access to the provided framework $B E E x$ (see Section 4) and used it to model their business ecosystem of focus. Thus, we were able to observe documentation during the entire study period. Both companies provided the researchers access to existing company material, which contributed to a shared understanding of the addressed business ecosystems and were used as data sources. Finally, we conducted several workshops in both studies with a high degree of interaction and made direct observations. Each workshop was documented in form of a report, which was validated with company representatives.

Data Analysis. The study aims at identifying how the ecosystem data model and ecosystem view model were instantiated and how they evolved following a collaborative modelling approach. After each workshop session, the direct observation records were analysed and the model created or adapted within this session. For both studies final workshops with a larger group of stakeholders - including for both the respective gatekeeper - were conducted in which both the status-quo of the created model and resulting visualization were used to gain insights on the business ecosystems.

The final two process steps - cross-contextual conclusion and implications - are included in the following sections.

\section{Business Ecosystem Explorer (BEEx)}

In the following, we describe the developed framework to collaboratively model and then visualize business ecosystems.

The Hybrid Wiki Approach to Collaborative Work. To address the dynamic structure of business ecosystems, we design an agile framework for modelling ecosystems as integrated, adaptive collaborative work system supporting the evolution of both the model and its instances at runtime by stakeholders and ecosystem experts (i.e., users without programming knowledge or skills). This framework rests on the Hybrid Wiki approach as presented in (Reschenhofer et al. 2016) that serves as 
Knowledge Management System application development platform and contains features for data management as well as collaboration and decision support. All changes can be traced back to the user responsible for the changes, including the time of the change. To create the business ecosystem model we use the Hybrid Wiki metamodel.

The Hybrid Wiki metamodel contains the following model building blocks: Workspace, Entity, EntityType, Attribute, and AttributeDefinition. These concepts structure the model inside a Workspace and capture its current snapshot in a data-driven process (i.e., bottom-up process). An Entity contains a collection of Attributes, and the Attributes are stored as a key-value pair. The Attributes have a name and can store multiple values of different types, for example, strings or references to other Entities. The user can create an Attribute at run-time to capture structured information about an Entity. An EntityType allows users to refer to a collection of similar Entities, e.g., organizations, persons. The EntityType consists of multiple AttributeDefinitions, which in turn contain multiple validators such as multiplicity validator, string value validator, and link value validator. Additionally, an Attribute and its values can be associated with validators for maintaining integrity constraints.

Business Ecosystem Explorer Model. The agile framework relies on a) ecosystem data model, and b) ecosystem view model, each with respective features for creation and adaption. Both models are encoded using the Hybrid Wiki metamodel.

The ecosystem data model contains the EntityTypes of relevance for the business ecosystem in focus. The view model is encoded as one EntityType called visualizations. Each visualization has two elements: the first element is the link between the data model and the visualizations. The second element is the specification of the visualizations using a declarative language. The proposed approach provides the feature of adapting the models at runtime. In case of chances in the data model, such as adding a category of entities, changing or deleting existing categories, the visualizations are updated at runtime.

Business Ecosystem Explorer Views. The agile framework - used as a basis for the studies currently consists of six views; a landing page, a list of all entities, detail view with company information, a relation view, a visualization overview, and several visualizations. For all views, a menu bar at the top of the page provides links to the other views available.

Within the studies, the framework was used to discuss the initial idea of business ecosystem modelling and visualization. At a later stage of the study, stakeholders of both enterprises used the framework to create their own workspace and selected appropriate visualizations.

\section{Case Studies: Collaborative Business Ecosystem Modelling}

For both studies we followed the approach to initiate modelling and visualizing of business ecosystems as illustrated in Figure 2. The process consists of overall six steps.

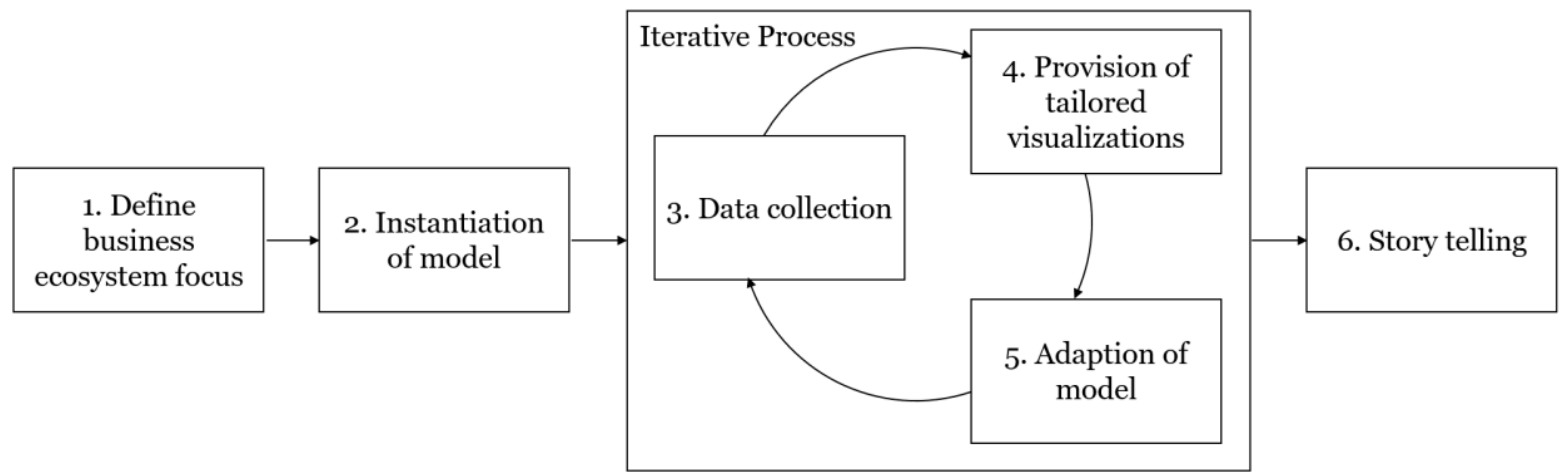

Figure 2: Approach to collaborative ecosystem modelling (adapted from Basole et al. (2016))

In the first step, the business ecosystem focus is defined. Examples for a business ecosystem focus are ecosystems established all around a technology platform, an ecosystem of a specific market exploiting specific digital technologies (Sako 2018) or ecosystems around one focal firm.

In the second step, initial models are created for both the data and the view model. Within the data model instantiation, the relevant entities of the ecosystem are defined, including attributes describing them. In addition, relation types between identified entities are preliminarily set. For the view model, the type of visualization including the specifications for this visualization are established. The requirements for both models should be collected - even in the instantiation phase of the models -by several stakeholders to ensure tailored visualizations in a later phase of the process. 
Third, a process consisting of the data collection step, to gather data about the ecosystem according to the specified data model, the provision of tailored visualization according to the view model and the adaption steps in which both models are modified using feedback collected from involved stakeholders. This three-step process is conducted iteratively until the collected data and the tailored visualization fulfil the stakeholders' requirements and needs.

In the final process step, the created visualizations are used to extract knowledge about the ecosystem, which contributes to a better understanding of the ecosystem in focus.

We used the BEEx framework to instantiate the business ecosystem model for both studies. That is why for both studies prior to the actual modelling workshops, a dedicated workshop to explain the BEEx framework was conducted, explaining the concept of data and view model and the adaption in run-time. Within these workshops, the aims for both studies were set: Achieving an instance of the business ecosystem model of focus and using the framework to initiate the management of the model evolution by following the process described in Figure 2.

As our focus was on the initiation of the company-internal business ecosystem modelling and visualization, the iteration of process steps three to five was only conducted a limited number of times for both studies.

Through the usage of the Knowledge Management System, we were able to identify the contributions of different involved stakeholders both during the workshops through observations but also using the provided collaboration feature. In the beginning of the studies, each stakeholder received a dedicated user identity. Thereby, in case of changes of the models in between workshops, these were transparent to the researchers.

During the studies, we identified roles of the group model building, which were involved in different process steps. Here we report about the process applied, the roles identified and their influence in the modelling results.

\subsection{Study 1: Innovative Mobility Services Business Ecosystem}

The first study together with an automotive company took place over five months (December 2017 to April 2018). Within the entire study time, overall five representatives were involved, whereby two of these five were active in the modelling workshop. The three other stakeholder were included in major decisions. The action research consists of 11 workshops each lasting between 60 and 120 minutes. All workshops happened on the enterprise premise using laptops, a whiteboard and pen and paper.

Course of the Study. The definition of the business ecosystem focus was set prior to the first modelling workshop of the study happening after the organizational workshop. Purpose of this study was to model and visualize the business ecosystem of innovative mobility services. Of interest, a better understanding about which cities are providing innovative mobility services within their mobility offer landscape and which service might be relevant for a city allocating currently less mobility services. Also of interest was a better understanding of which service provider offers which mobility services and how well each service provider is interconnected with which city. Thereby, the business ecosystem of focus was superficially analysed prior to the conduction of the study. Information about the business ecosystem was collected by several company's stakeholders and documented in an unstructured form. The information was not processed any further, a tracking about who included which information and who accessed it in a later stage was not conducted, and no visualization was used.

Within the first three modelling sessions (December and January), the initial data model was created. As the Knowledge Management System within the BEEx framework provides the feature of supporting the evolution of models, the data model was in the following continuously updated and adapted.

In the following two months, the two company representatives implemented the already within the company collected data in the system and enriched it with additional data. Therefore, company external data sources such as newspaper articles, news feeds and free of charge online databases were used. The data collection process was conducted manually by enterprise representatives and not supported by the researchers. During this phase, four modelling workshops were conducted. Within these workshops, the involved researchers answered question about the usage of the Knowledge Management System. In addition, inconsistencies of the model were addressed and solved.

Simultaneously in three workshops in February and March, the view model was created and the initial visualization type defined: a force-layout view. This decision happened after two months of business ecosystem data modelling and data collection. In a dedicated workshop, the building blocks (marks, scales, and signals) of each visualization were discussed and aligned. After this aligned view model, the 
researched created the visualizations accordingly. The tailored view were presented and discussed in the following workshops end of March. Collecting the feedback, the visualizations were adapted and further discussed in two workshops in April.

The final workshop happened end of April. Besides the gatekeeper, the two modellers and the two researchers, two additional enterprise stakeholders participated. The results of the previous modelling workshops were presented: Besides the two force layout views displayed in Figure 3, a list view of all entities, a detailed view for each entity and one additional force-layout view were presented and discussed. The feedback of the gatekeeper and additional modeller regarding the view model were incorporated immediately.

Data and View Model. The Hybrid Wiki metamodel was used to set up the data and view model. After the first data model creation workshop, the data model consisted of six EntityTypes and eight AttributeDefinitions. In the following session, the data model grew to finally eight EntityTypes and 26 AttributeDefinitions, a greater increase on the attribute level than on the entity level. As entities of the ecosystem, cities, organizations (e.g., services providers such as automotive OEMs), mobility services and mobility related projects were modelled. For example, the services comprised car sharing, bike sharing, ride sharing, to name just a few.

As described in Section 4, the view model consisted of the three entities describing the three force layouts. Two of the three views as visualized in Figure 3.

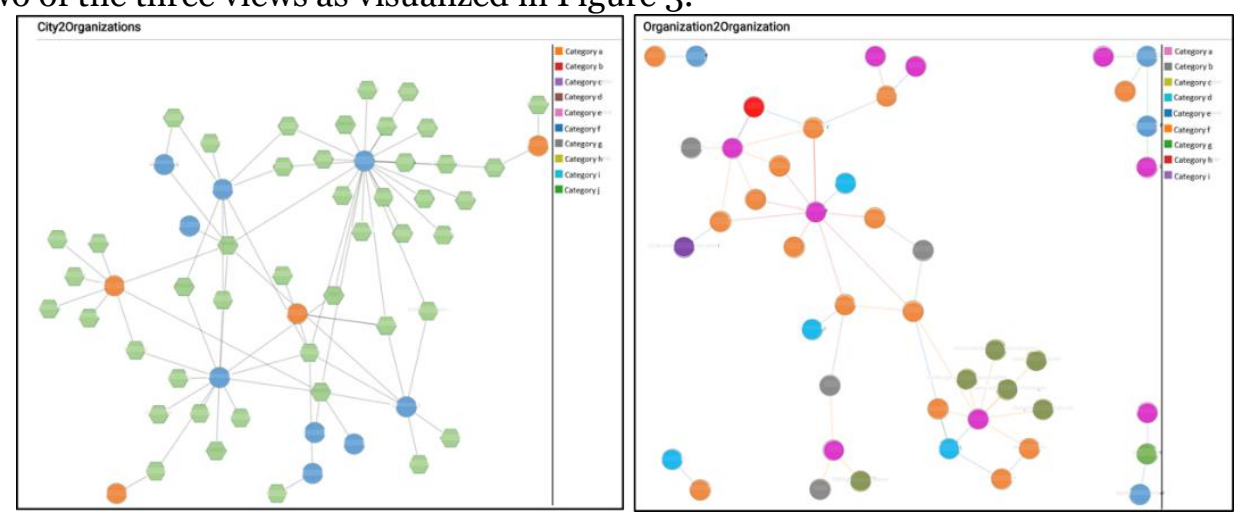

Figure 3: Two visualization created in the first study. Due to anonymization requirements, the entity names and categories are blurred.

Roles of the Collaborative Process. We validated four roles in this study: facilitator, modeller, recorder and gatekeeper. The gatekeeper was the most prominent participant in the beginning of the study. She decided on the business ecosystem focus and assigned stakeholders to participate in the modelling sessions. Within the second process step, one company representative established herself as the facilitator. She paid constant attention to the group process. She also took the lead in organizing all following modelling sessions. The facilitator was also the leading contributor in implementing existing data, enriching it with external data sources and thereby acting as a modeller especially between workshops. During this final workshop, she again took the lead explaining the results and the outcome.

The researchers present in the workshops fulfilled both the modeller and recorder role by documenting each workshop in form of a written report.

\subsection{Study 2: Publishing Company Business Ecosystem}

The second study was conducted with a publishing company. The study took place over six months (February to July). Overall, six enterprise representatives participated in the study in seven workshops each lasting between 90 and 120 minutes. All seven workshops took place on the enterprise premise using laptops, a whiteboard and pen and paper.

Course of the Study. Within the first workshop in February, the focus of the business ecosystem was set: German publishing business ecosystem with regard to key persons, publishing landscape and the services offered and consumed. Besides identifying the ecosystem focus, previous activities and relevant additional stakeholder to involve were discussed. Information of the ecosystem in focus had yet not been analysed and the ecosystem not yet modelled. A group of potential relevant stakeholder to contribute to the modelling process were identified.

In the next two workshops, the data model was aligned. The second workshop thereby contributed to enrichment of the data model, focusing on attributes for each ecosystem entity. 
In a workshop in April, the view model including the building blocks was discussed and aligned.

For the next two workshops in May, two visualizations were provided by the researchers using the data implemented in the Knowledge Management System. Thereby, both company internal data sources but also news articles were used.

After adapting the models according to the discussion, in an additional workshop in May three further company representatives were invited to discuss the initially now available tailored instance of the $B E E x$ framework. The visualizations are displayed in Figure 4. In addition to these two visualizations, a customized landing page was created.

Data and View Model. Within the study both the data and view model according to the previous described Hybrid Wiki metamodel were created. After the first data model creation workshop, three EntityTypes with seven AttributeDefinitions were defined. At the end of the study, the final data model consisted of nine EntityTypes. These EntityTypes included 24 AttributeDefinitions. The data model evolved during the modelling process, especially on the attribute level and during the initial phase of the project. For the data model, ecosystem entities such as key persons, publisher, and publishing groups were identified, including relation types such as role of the person within the ecosystem and type of service. The view model consisted of the two entities covering the visualizations displayed in Figure 4. After the visualizations were created, the data model on the entity level stayed the same.

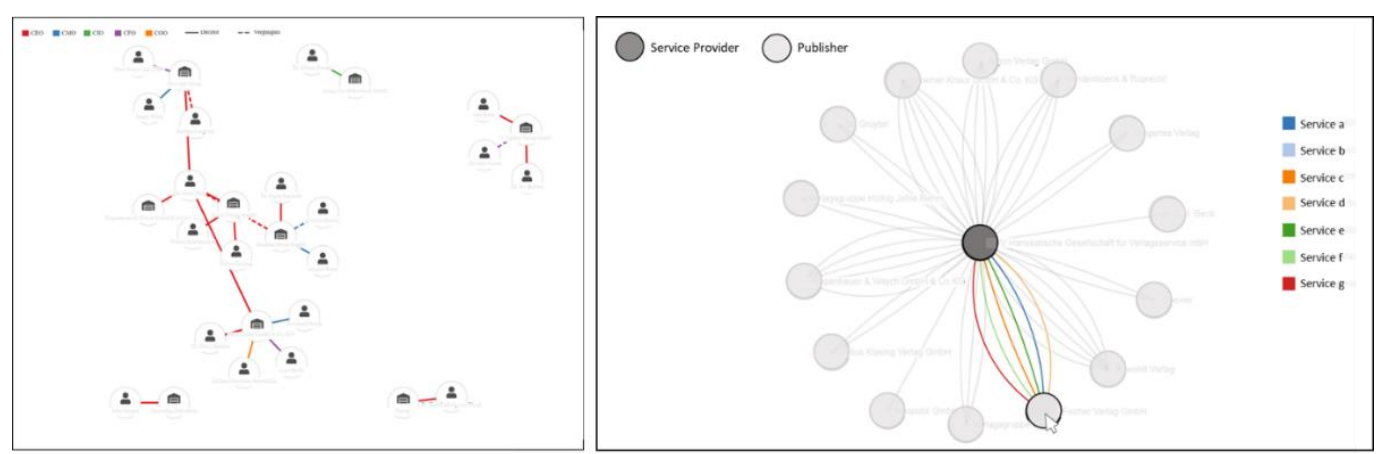

Figure 4: Two visualization created in the second study. Due to anonymization requirements, the entity names and categories are blurred.

Roles of the Collaborative Process. Similar to the first study, we could validate four roles in this study: facilitator, modeller, recorder and gatekeeper. Within this study, the composition of the modelling groups changed within the study period. All participants, which actively modelled, participated in the pre-study workshop and thus were familiar with the BEEx framework and the study aim. For all workshops, the group consisted of at least three participants.

Already in the first workshop, one of the company's representatives positioned herself as the facilitator. She kept this role over the entire study period and participated in all workshops. The facilitator also actively participated in the modelling as such, but acted as decision maker and discussion leader. One example is the decision to use a force-layout. The gatekeeper only participated twice in workshops in May and thus was identified at a late state of the process. Even though, not included in the previous workshops, her opinion and feedback to the provided and discussed visualizations were high prioritized by the facilitator. The researcher present in the workshops fulfilled the role of the recorder and documented each workshop in form of a written report.

\section{Cross-case Conclusions: Findings and Lessons Learned}

Even though in both studies different topics and ecosystem models have been instantiated, we can draw some cross-case conclusions. Following Patton (2001) who defines lessons learned as the knowledge which is derived from the screening of a situation and which can be applied in similar situations in the future, we will present our finding and lessons learned of both studies.

Evolution of the Data Model. We noticed for both case studies, that the data model evolved rather on the AttributeType Level than on the EntityType Level. That means, the entities visualized remained nearly untouched whereby for all entities the attributes changed during the modelling process. In our opinion, this is due to the user being accustomed to the provided visualization and thus is less willing to change it. A change of the attribute, which might also include adding or deleting an attribute, does not 
involve changes of the force-layout view. Furthermore, as the attributes can be defined as mandatory attributes or not, the user is free in adding attributes for specific entities that are not available for others.

Expertise of Modelling Participants. Comparing both studies we noticed that the existing knowledge of the workshop participants has a great influence in the achieved results. The modellers involved in the first study were less experienced with modelling activities as such. One consequence was more time spent on the data and view model creation and thus receiving the implemented interactive visualizations at a later point within the process. To address this, an additional workshop prior to the study period focussing on how to create a model is recommendable. Our pre-study workshop solely focused on explaining the existing framework and the data and view model in use there but not on active participation of the study participants.

Maintaining Motivation during Modelling Process. Especially in the first study we noticed a decrease in motivation during the data and view model creation phase. As described above, the study participants - including the facilitator - were unfamiliar with modelling activities. Thus, reaching the fourth process step took longer than expected by the company representatives. This hold especially true, as the predefined outcome of the study was an instance of the BEEx framework including tailored interactive visualizations.

Importance to Provide Visualizations. The aforementioned findings led to the conclusion, that the visualizations play a crucial role in the perceived success of the modelling process. We noticed within both studies that as soon as the interactive visualizations were provided with the additional feature of adapting changes to the data model and the data collected in run-time contributed heavily to motivate the participants to collect and implement data.

Story Telling. As for both studies the focus was on the instantiation of the ecosystem model, the provided visualizations can be considered as early visualizations of the ecosystems. Within the studies, this process step was rather used to present the results of the study to the gatekeeper.

Collaborative Modelling. In both studies, several stakeholders were included in the modelling process. During the workshops, all participants contributed as modellers. Between the workshops, the overall contribution decreased, and only key team members, those we identified as facilitators, continued collecting and implementing data. Changes of the model were only implemented during the workshops, which might be due to the still rather unfamiliar framework in use. We recognized a lively discussion during the workshops, implying the relevance of managing the business ecosystem model in focus.

We identified four clear roles within both studies: The facilitator, the modeller, the recorder and the gatekeeper. As we conducted this process of instantiating a business ecosystem model first time, a process coach was missing.

\section{Discussion and Conclusion}

In this paper, we presented a process to model and visualize business ecosystems from a company internal perspective, which we applied in two company settings. This process supports several stakeholders to collaboratively contribute to the instantiation of a business ecosystem model.

The process consists of six steps, namely, the definition of the business ecosystem in focus, the instantiation of both the data and view model, the collection of relevant data, the provision of tailored visualization, feedback, which is fed into the iteration process of the two previous steps, and finally, the story-telling step. We report in detail about the study development, the involvement of participants and conclude with findings and lessons learned.

A noticeable limitation of the presented work is the usage of only manually implemented data, which is a time-consuming and tedious work. As visualizations are data-driven, the business ecosystem visualizations rely heavily on the availability and quality of data. Complementing the manual data collection with (semi-)automated data extraction process steps, such as provided with Natural Language Processing, could contribute to the availability of data and thus to richer visualization leading to improve possibilities to extract knowledge. For the quality of data, approaches to data governance are missing in the process presented. We envision the facilitator being a good starting point for such a role.

In addition, as this work focuses on the instantiation of an ecosystem modelling, we envision the iterative process steps to be conducted several time in future research. In our view this would lead to data enrichment and potential inclusion of additional stakeholders within the company. 
As a major challenge, we realized the right balance between early providing tailored visualizations and following the process. As our focus was to establish a shared language within the modelling process, we first provided visualization after aligning the data and the view model, leading to a motivational decrease during the study. Nevertheless, we believe the presented process can be used as a reference by organizations interested in modelling their business ecosystem.

\section{References}

Basole, R. C. 2009a. "Structural Analysis and Visualization of Ecosystems : A Study of Mobile Device Platforms," Proceedings of the Fifteenth Americas Conference on Information Systems San Fransisco California, August, pp 1-10.

Basole, R. C. 2009b. "Visualization of interfirm relations in a converging mobile ecosystem," Journal of Information Technology (24:2), pp 144-159.

Basole, R. C., Huhtamäki, J., Still, K., and Russell, M. G. 2016. "Visual decision support for business ecosystem analysis," Expert Systems with Applications, (65), pp 271-282.

Basole, R. C., Russell, M. G., Huhtamäki, J., and Rubens, N. 2012. "Understanding Mobile Ecosystem Dynamics: A Data-Driven Approach," International Conference on Mobile Business, (15) http://aisel.aisnet.org/icmb2012/15/ Retrieved: 12 July 2018.

Basole, R. C., Russell, M. G., Huhtamäki, J., Rubens, N., Still, K., and Park, H. 2015. "Understanding Business Ecosystem Dynamics: A Data-Driven Approach," ACM Transactions on Management Information Systems (6:2), June, pp 1-32.

Bosch, J. 2016 "Speed, Data, and Ecosystems: The Future of Software Engineering," IEEE Software (33:1), January, pp 82-88.

Benbasat, I., Goldstein, D. K., and Mead, M. 1987. "The Case Research Strategy in Studies of Information Systems Case Research,” MIS Quarterly (11:3), pp 369-386.

Dollmann, T., Houy, C., Fettke, P., and Loos, P. 2011. "Collaborative business process modeling with CoMoMod: A toolkit for model integration in distributed cooperation environments," Proceedings of the 2Oth IEEE International Workshops on Enabling Technologies: Infrastructure for Collaborative Enterprises, pp 217-222.

Evans, P. C., and Basole, R. C. 2016. "Revealing the API ecosystem and enterprise strategy via visual analytics," Communications of the ACM (59:2), pp 26-28.

Faber, A., Hernandez-Mendez, A., Rehm, S.-V., and Matthes, F. 2018. "An Agile Framework for Modeling Smart City Business Ecosystems," Proceedings of the 2oth International Conference on Enterprise Information Systems (2), pp 39-50.

Guittard, C., Schenk, E., and T. Burger-Helmchen, T. 2015. "Crowdsourcing and the Evolution of a Business Ecosystem,” in Advances in Crowdsourcing, F. J. Garrigos-Simon, I. and Gil-Pechuàn, and S. and Estelles-Miguel, Eds. Springer International Publishing, pp 49-62.

Hao, J., Zhu, J., and Zhong, R. 2015. "The rise of big data on urban studies and planning practices in China: Review and open research issues," Journal of Urban Management (4:2), pp 92-124.

Huhtamaki, J., and Rubens, N. 2016. "Exploring innovation ecosystems as networks: Four european cases," Proceedings of the Annual Hawaii International Conference on System Sciences, March, pp 4505-4514.

Iansiti, M., and Levien, R. 2004. "Strategy as Ecology," Harvard Business Review (82:3), https://hbr.org/2004/03/strategy-as-ecology Retrieved: 12 July 2018.

Iyer, B. R., and Basole, R. C. 2016. "Visualization to understand ecosystems," Communications of the $A C M$ (59:11), pp 27-30.

Leonardi, P. M. 2011. "When Flexible Routines Meet Flexible Technologies: Affordance, Constraint, and the Imbrication of Human and Material Agencies," MIS Quarterly (35:1), pp 147-167.

Liu, F., and Zhang, C. 2010. "Role-based collaborative model of group decision support system," Seventh International Conference on Fuzzy Systems and Knowledge Discovery, pp 1039-1043.

Moore, J. F. 1996. The Death of Competition: Leadership and Strategy in the Age of Business Ecosystems. New York: HarperBusiness. 
Park H., and Basole, R. C. 2016. "Bicentric diagrams: Design and applications of a graph-based relational set visualization technique," Decision Support Systems (84), April, pp 64-77.

Park, H., Bellamy, M. A., and Basole, R. C. 2016. "Visual analytics for supply network management: System design and evaluation,” Decision Support Systems (91), pp 89-102.

Patton, M. Q. 2001. "Evaluation, knowledge management, best practices, and high quality lessons learned," The American Journal of Evaluation (22:3), pp 329-336.

Peltoniemi M., and Vuori, E. 2004. "Business ecosystem as the new approach to complex adaptive business environments," Proceedings of eBusiness Research Forum, pp 267-281.

Porter, M. 1979. "How competitive forces shape strategy," Harvard Business Review, March, pp 102117.

Rehm, S.-V., Faber, A., and Goel, L. 2017. "Visualizing Platform Hubs of Smart City Mobility Business Ecosystems," Proceedings of the Thirty Eighth Interantional Conference on Information Systems, pp 1-10.

Renger, M., Kolfschoten, G. L., and De Vreede, G. J. 2008. "Challenges in collaborative modeling: A literature review," Lecture Notes in Business Information Processing (10:10), pp 61-77.

Reschenhofer, T., Bhat, M., Hernandez-Mendez, A., and Matthes, F. 2016. "Lessons learned in aligning data and model evolution in collaborative information systems," Proceedings of the 38th International Conference on Software Engineering Companion, pp 132-141.

Richardson, G. P., and Andersen, D. F. 1995. “Teamwork in group model building,” System Dynamics Review (11:2), pp 113-137.

Roth, S., Hauder, M., and Matthes, F. 2013. "Collaborative evolution of enterprise architecture models,” CEUR Workshop Proceedings (1079), pp 1-12.

Sako, M. 2018. "Business Ecosystems: How Do They Matter for Innovation?," Communications of the $A C M$ (61:4), March, pp 20-22.

Uchihira, N., Ishimatsu, H., and Inoue, K. 2016. "IoT service business ecosystem design in a global, competitive, and collaborative environment," Portland International Conference on Management of Engineering and Technology (PICMET), pp 1195-1201.

Vartak, M., Huang, S., Siddiqui, T., Madden, S., and Parameswaran, A. 2017. "Towards Visualization Recommendation Systems," ACM SIGMOD Record (45:4), May, pp 34-39.

Visnjic, I., Neely, A., Cennamo, C., and Visnjic, N. 2016. “Governing the City,” California Management Review (59:1), pp 109-140.

Yin, R. K. 1994. "Case study research: Design and Methods," Applied social research methods series (5), Sage Publications, London.

\section{Acknowledgements}

This work has been sponsored by the German Federal Ministry of Education and Research (BMBF) grant BEEx+ 01IS17049. This work is further part of the TUM Living Lab Connected Mobility (TUM LLCM) project and has been funded by the Bavarian Ministry of Economic Affairs, Energy and Technology (StMWi) through the Center Digitisation.Bavaria, an initiative of the Bavarian State Government

\section{Copyright}

The following copyright paragraph must be appended to the paper. Author names MUST not be included until after reviewing. Please ensure the hyperlink remains for electronic harvesting of copyright restrictions.

Copyright: (C) 2018 authors. This is an open-access article distributed under the terms of the Creative Commons Attribution-NonCommercial 3.0 Australia License, which permits non-commercial use, distribution, and reproduction in any medium, provided the original author and ACIS are credited. 